\title{
Temporal Spectrum of a Scattered Electromagnetic Waves in the Conductive Collision Turbulent Magnetized Plasma
}

\author{
G. Jandieri', A. Ishimaru², B. Rawat 3 , and N. Tugushi ${ }^{4}$ \\ ${ }^{1}$ International Space Agency Georgian Society, GTU, Tbilisi, 0160, Georgia \\ ${ }^{2}$ Department of Electrical and Computer Engineering, University of Washington, Seattle, WA, 98195, USA \\ ${ }^{3}$ Department of Electrical and Biomedical Engineering, University of Nevada, Reno, NV, 89557-0260, USA \\ ${ }^{4}$ Department of Physics, Tbilisi State University, I. Chavchavadze av., 1, Tbilisi, 0128, Georgia \\ Corresponding author: N. Tugushi (e-mail: tugushinika96@gmail.com).
}

\begin{abstract}
Using WKB method the peculiarities of the temporal spectrum of an ordinary and extraordinary electromagnetic wave scattered in a weakly randomly inhomogeneous three-dimensional nonstationary and conductive magnetized plasma are investigated. On the basis of the stochastic differential transport equation for the frequency fluctuation the broadening and the displacement of the temporal spectrum for both waves are obtained for the polar terrestrial ionosphere. These statistical characteristics contain anisotropic parameters: velocity of a plasma stream, conductivities of the ionosphere, elongated electron density irregularities are characterized by the anisotropy factor and inclination angle with respect to the geomagnetic lines of forces. The analysis of the power spectrum of the waves as a function of the propagation distance and the nondimensional frequency parameter containing the carrier frequency and characteristic temporal scale of electron density fluctuations are carried out. Analytical and numerical calculations have shown that the terrestrial conductivity and anisotropy factors exert a substantial influence on evaluation of the temporal spectrum than the inclination angle. It was found that the wave spectrum increases initially as the square root of the propagation distance, but at large distances it approaches a limiting value. Statistical moments of a scattered ordinary and extraordinary waves do not depend on an absorption sign and valid for both absorptive and active media.
\end{abstract}

INDEX TERMS Statistical moments, temporal spectrum, turbulent magnetized plasma.

\section{INTRODUCTION}

A $\mathrm{S}$ is well-known, turbulent media are characterized by macroscopic motion, chaotical nonstationarity and inhomogeneity. Statistical characteristics of waves in random media with randomly varying spatial irregularities are studied well [1-4] but immediate action of the movement of the environment and its nonstationarity on the statistical characteristics of scattered waves is not studied in detail. Nonstationarity may be substantial and cause changes of properties of the considered waves. Meanwhile, the effect of accumulation of fluctuations of wave parameters is revealed more brightly at big distances from a source at existence of regular absorption in the nonstationary medium [5].

The problem is of importance since the performance of various existing and developing communication systems depend on the statistical properties of the signals after propagating through the transmission medium. In most cases using results derived applying geometrical optics and the parabolic approximation. This imposes any restrictions on the distance travelling by wave in random medium. Broadening and shift of maximum of the spatial power spectrum of scattered electromagnetic waves in the geometrical optics approximation were considered in [6-9].

Evaluation of the temporal spectrum of an incident narrow band wave field in a nonstationary medium is characterized by both amplitude and frequency modulation. Transport theory to study frequency spectra of beam waves propagating in a turbulent atmosphere was used in [10]. Spectral broadening has also been considered in [11]. Their investigation was based on the parabolic approximation with "frozen" turbulence model [12]. The behavior of the temporal (frequency) spectrum of multiple scattered electromagnetic waves in the collisionless turbulent nonmagnetized plasma has been investigated in $[13,14]$. Nonstationarity is caused due to plasma motion. It was shown that power spectrum of wave has the Gaussian shape with the displaced maximum. Width of the spectrum coincides with variance of the frequency fluctuation calculated in the geometrical optics approximation by the perturbation method.

In many papers the fluctuating medium is considered as nonabsorptive. The influence of absorption (conductivity of 
plasma and collision between plasma particles) on the wave parameters fluctuations in the magnetized turbulent plasma is important.

Broadening of the frequency spectrum characterize wave propagation in a time-dependent plasma, and is a result of frequency modulation of the spectral components of the wave by the time variation in the properties of turbulent plasma.

This paper addresses propagation of the ordinary $(\mathrm{O}$ wave) and extraordinary ( $\mathrm{E}$ - wave) electromagnetic waves in the polar region of the terrestrial ionosphere; transformation of the temporal spectrum in the conductive collision magnetized ionospheric plasma with slowly varying spatial-temporal electron density fluctuations using the geometrical optics approximation. We present and evaluate the performance of a processing approach to estimate the parameters for the temporal spectrum of the turbulent magnetized collision plasma. Evaluation of the temporal spectrum depends on the following anisotropy parameters: direction of an external magnetic field, plasma velocity, anisotropy of the ionospheric conductivities, anisotropy factor (the ratio of elongated and transversal characteristic spatial scales of plasmonic structures) and inclination angle of ionospheric anisotropic electron density irregularities with respect to the external magnetic field. In section 2, stochastic transport equation for the frequency fluctuation is derived from the eikonal equation. This equation includes the effects of multiple scattering in medium which vary with both position and time. In section 3 , these statistical characteristics are computed for the anisotropic Gaussian correlation function of electron density fluctuations containing parameters of the anisotropic plasmonic structures and the velocity of plasma motion.

Analytical and numerical calculations have been carried out for the anisotropic Gaussian spatial-temporal spectrum of electron density fluctuations applying the experimental data. Numerical calculations are presented for typical ionospheric irregularities. Some conclusions and discussion are given in Section 4.

\section{FORMULATION OF THE PROBLEM}

At the present time the most developed and proved method description of short $(2-40 \mathrm{MHz})$ radio waves propagation in the terrestrial ionosphere as theoretically as well as experimentally is the four-dimensional WKB (WentzelKramers-Brillouin) method. This method approximately describes wave fields in a smoothly-inhomogeneous media [1-3]. The postulate on representation of the field as fields of locally plane wave is $\mathbf{E}(\mathbf{r}, t)=\tilde{\mathbf{E}}(\mathbf{r}, t) \exp [i \varphi(\mathbf{r}, t)]$, where the amplitude $\tilde{\mathbf{E}}(\mathbf{r}, t)$ is a slowly varying function, $\varphi(\mathbf{r}, t)$ is a complex phase. In the conductive collision turbulent magnetized plasma, the complex index of refraction $N(\mathbf{r}, t)=N_{0}(\mathbf{r}, t)-i N_{1}(\mathbf{r}, t)$, contains the electron density $n(\mathbf{r}, t)=n_{0}+n_{1}(\mathbf{r}, t)$ depending on the spatial coordinates and time. The first term $n_{0}$ is a constant value, the second one is a random function of the spatial coordinates and time, however $\left|n_{1}\right|<<n_{0}$. In a zero-order approximation of the geometrical optics, neglecting the depolarization of the electric field we obtain the eikonal equation $c^{2} k^{2}=\omega^{2} N(\omega, n)$, where $\omega(\mathbf{r}, t)=\partial \varphi / \partial t$ is the frequency and $\mathbf{k}(\mathbf{r}, t)=-\nabla \varphi$ is the wave vector, $c$ is the speed of light. This equation determines the virtual ray trajectories on which integrated characteristics of the field which can be experimentally measured are calculated.

At first, we will consider behavior of the virtual beam trajectories caused not absorbing uniform plasma with the refractive index $N=$ const . For a plane monochromatic wave, as is well-known, $u=A \exp (-i k N z)$, where the amplitude $A$ and the phase $\Phi$ are real quantities. Poynting vector $\mathbf{P}$ in a zero-order approximation of the geometrical optics is parallel to a vector $\nabla \Phi$ and directed on a normal to the phase front. In the absorbing plasma, for almost plane wave there are two non-coincidence directions: $\nabla \Phi_{1}$ (is the direction of the phase propagation) and $\nabla \Phi_{2}$ (is the fast-dumping direction of the wave). The term "almost plane wave" is justified by the fact that in the area of the order of $2 \pi / k N$ the field is the same as in nonabsorbing media. In this case wave fronts are not flat, and the normals to the wave fronts are not parallel, but are close to parallel.

Neglecting polarization effects Poynting vector can be written as $\mathbf{P}=c \mathbf{I} / 8 \pi$ [3]. In this case average on time vector of the energy flux density is equal to

$$
\mathbf{P}=\frac{c}{8 \pi k} \operatorname{Im}\left(E^{*} \nabla E\right)
$$

Substituting here $E=A \exp (i k \Phi)$, where $A=A_{1}+i A_{2}$, $\Phi=\Phi_{1}+i \Phi_{2}$, we obtain:

$$
\mathbf{I}=\exp \left(-2 k \Phi_{2}\right)\left\{|A|^{2} \nabla \Phi_{1}+\frac{A_{1}^{2}}{k_{0}} \nabla\left(\frac{A_{2}}{A_{1}}\right)\right\}
$$

It's clear that wave energy is propagate in the direction $\nabla \Phi_{1}$, i.e., normal to the surfaces $\Phi_{1}=$ const, and exponentially attenuates with growth $\Phi_{2}$, and the direction of the maximum attenuation is defined by a vector $\nabla \Phi_{2}$ (this can be shown, having taken a gradient from $|\mathbf{I}|$ ). In the absorptive (collisional and conductive) plasma virtual ray trajectories are bent and deviate towards increase of the refractive index $N$, i.e., towards to the vector $\nabla N$. The ray does not change its direction of propagation in media which vary only with time.

Frequency can be defined as the time derivative of the phase function of both the position and the time at which 
the observation is made. Now calculate statistical characteristics of scattered electromagnetic waves in an absorptive plasma. Under the usual assumptions of neglecting the depolarization of the field, in the spatialtemporal geometrical optics approximation wave frequency satisfies the stochastic differential transport equation $[5,15]$ :

$$
\frac{\partial \omega}{\partial t}+\left(\mathbf{u}_{g r} \nabla \omega\right)=-\omega\left[\frac{\partial(N \omega)}{\partial \omega}\right]^{-1} \frac{\partial N}{\partial n} \frac{\partial n}{\partial t},
$$

where $u_{g r}=c[\partial(N \omega) / \partial \omega]^{-1}$ is the group velocity.

Complex index of refraction of the conductive turbulent collision magnetized plasma in the polar region of the terrestrial atmosphere is [16]:

$$
N(n, \omega)=N_{0}\left(n_{0}, \omega\right)-i N_{1}(n, \omega),
$$

where: $N_{0}=\sqrt{\left(r+\mathrm{T}_{0}\right) / 2}, \quad N_{1}=\sqrt{\left(r-\mathrm{T}_{0}\right) / 2}$,

$$
\begin{aligned}
& r=\sqrt{\mathrm{T}_{0}^{2}+\mathrm{T}_{1}^{2}}, \mathrm{~T}_{0}=\left[A_{0}\left(B_{0} \pm G_{0}\right)-A^{\prime}\left(-B^{\prime} \pm G^{\prime}\right)\right], \\
& \mathrm{T}_{1}=A^{\prime}\left(B_{0} \pm G_{0}\right)+A_{0}\left(-B^{\prime} \pm G^{\prime}\right), G_{0}=\sqrt{\left(r+D_{0}\right) / 2}, \\
& G^{\prime}=\sqrt{\left(r-D_{0}\right) / 2}, \quad r=\sqrt{D_{0}^{2}+D^{\prime 2}}, D_{0}=B_{0}^{2}-B^{\prime 2}- \\
& -4\left(A_{0} C_{0}-A^{\prime} C^{\prime}\right), D^{\prime}=-2 B_{0} B^{\prime}+4\left(A_{0} C^{\prime}+C_{0} A^{\prime}\right) ; \\
& A_{0}=\varepsilon_{\perp} \sin ^{2} \theta+\varepsilon_{\|} \cos ^{2} \theta, A^{\prime}=\sigma_{\perp} \sin ^{2} \theta+\sigma_{\|} \cos ^{2} \theta ; \\
& B_{0}=\left(\varepsilon_{\perp} \varepsilon_{\|}-\sigma_{\perp} \sigma_{\|}\right)\left(1+\cos ^{2} \theta\right)+\left[\left(\varepsilon_{\perp}^{2}-\sigma_{\perp}^{2}\right)-\left(\mathfrak{x}+\sigma_{H}\right)^{2}\right] \\
& \cdot \sin ^{2} \theta, B^{\prime}=\left(\varepsilon_{\perp} \sigma_{\|}+\varepsilon_{\|} \sigma_{\perp}\right)\left(1+\cos ^{2} \theta\right)+2 \varepsilon_{\perp} \sigma_{\perp} \sin ^{2} \theta, \\
& C^{\prime}=2 \varepsilon_{\|} \varepsilon_{\perp} \sigma_{\perp}+\sigma_{\|}\left[\varepsilon_{\perp}^{2}-\sigma_{\perp}^{2}-\left(\mathfrak{x}+\sigma_{H}^{2}\right)\right], \\
& C_{0}=\varepsilon_{\|}\left[\varepsilon_{\perp}^{2}-\sigma_{\perp}^{2}-\left(\mathfrak{x}+\sigma_{H}\right)^{2}\right]-2 \varepsilon_{\perp} \sigma_{\perp} \sigma_{\|} ;
\end{aligned}
$$

the dependence $N$ on the frequency means an account of a temporal dispersion; upper sign corresponds to the ordinary wave, lower sign - to the extraordinary wave, $\theta$ is the angle which the wave vector of a refracted wave in the magnetized plasma makes with the external magnetic field (along the Z-axis), YOZ is the main plane;

$$
\begin{aligned}
& \varepsilon_{\perp}=1-\mathrm{v} /(1-u), \varepsilon_{\|}=1-\mathrm{v}, \mathrm{v}(\mathbf{r}, t)=\omega_{p}^{2}(\mathbf{r}, t) / \omega^{2}, \\
& \mathfrak{x}=\mathrm{v} \sqrt{u} /(1-u), \mathrm{v}(\mathbf{r}, t)=\omega_{p}^{2}(\mathbf{r}, t) / \omega^{2} \text { and }
\end{aligned}
$$

$u=\left(e H_{0} / m_{e} c \omega\right)^{2}$ are magneto-ionic parameters of the ionospheric plasma, $\omega_{p}(\mathbf{r}, t)=\left[4 \pi N_{e}(\mathbf{r}, t) e^{2} / m_{e}\right]^{1 / 2}$ is the electron plasma frequency; $\tilde{\sigma}=4 \pi \hat{\sigma} / k_{0} c$ is the normalized conductivity having components:

$$
\begin{gathered}
\sigma_{\perp}=e^{2} n_{e}\left[\frac{v_{e}}{m_{e}\left(v_{e}^{2}+\omega_{e}^{2}\right)}+\frac{v_{i}}{m_{i}\left(v_{i n}^{2}+\omega_{i}^{2}\right)}\right], \\
\sigma_{H}=e^{2} n_{e}\left[\frac{\omega_{e}}{m_{e}\left(v_{e}^{2}+\omega_{e}^{2}\right)}-\frac{\omega_{i}}{m_{i}\left(v_{i n}^{2}+\omega_{i}^{2}\right)}\right], \\
\sigma_{\|}=e^{2} n_{e}\left(\frac{1}{m_{e} v_{e}}+\frac{1}{m_{m} v_{i n}}\right),
\end{gathered}
$$

$\sigma_{\perp}$ and $\sigma_{H}$ are the Pedersen and Hall's conductivities, respectively, $\sigma_{\|}$is the longitudinal (parallel to the magnetic field) conductivity; $e$ and $m_{e}$ are the charge and mass of an electron, $v_{e}=v_{e n}+v_{i n}$ is the effective collision frequency of electrons with other plasma particles, $\omega_{e}$ and $\omega_{i}$ are the angular gyrofrequencies of an electron and ion, respectively; the source irradiates a plane wave with the carrier frequency $\omega_{0}$.

In a homogeneous nonconductive plasma, the directions of the phase and group velocities of the ordinary and extraordinary electromagnetic waves coincide with the direction of an external magnetic field, while in conductive magnetized collision plasma these waves have the opposite directions. In the absorptive plasma the group velocity may be complex and may even have a magnitude greater than the speed of light, even in the limit of spatiallyhomogeneous, time invariant media.

Electromagnetic waves with different frequencies scattered on the spatial-temporal varying ionospheric irregularities, the receiving signal has the Doppler shift and the spectrum broadens. They are caused by electron density irregularities vary with both position and time. Now we will consider statistical moments of the wave frequency fluctuations, which are one of the important spectral characteristics specifying for nonstationary media. They can be found by measurements of a phase. Variance of the frequency fluctuations determines the width of a temporal power spectrum in the turbulent collision magnetized plasma using WKB methods.

In most cases involving wave propagation in real media such as the terrestrial ionosphere, the fluctuations in the plasma properties can be regarded as small. Thus, it is realistic, as well as mathematically convenient. We assume that in a zero-order approximation a plane wave propagates in the $z$ direction. The frequency fluctuation in the first order approximation satisfies the stochastic transport differential equation:

$$
\frac{\partial \omega_{1}}{\partial z}+\frac{m_{0}}{c}\left(1-i \Omega_{0}\right) \frac{\partial \omega_{1}}{\partial t}=-k_{0}\left(\Omega_{1}-i \Omega_{2}\right) \frac{\partial n_{1}}{\partial t},
$$

here: $\Omega_{0}=m_{1} / m_{0}, \quad m_{0}=N_{0}+\omega_{0}\left(\partial N_{0} / \partial \omega_{0}\right)$, $m_{1}=N_{1}+\omega_{0}\left(\partial N_{1} / \partial \omega_{0}\right), k_{0}=\omega_{0} / c$, 


$$
\begin{array}{r}
\Omega_{1}=\frac{1}{4 N_{0}}\left[\left(1+\frac{1}{r}\right) \frac{\partial T_{0}}{\partial n_{0}}+\frac{\partial T_{1}}{\partial n_{0}}\right], \\
\Omega_{2}=\frac{1}{4 N_{1}}\left[-\left(1+\frac{1}{r}\right) \frac{\partial T_{0}}{\partial n_{0}}+\frac{\partial T_{1}}{\partial n_{0}}\right] .
\end{array}
$$

Right part (3) contains electron density fluctuation $n_{1}$, a small parameter which is a measure of the magnitude of the fluctuations of the plasma with zero mean. Equation (3) has been derived in the assumption that an unperturbed wave is a plane monochromatic wave propagating along the $\mathrm{z}$-axis, $\varphi=\omega_{0} t-k_{0} z$; the group velocity of an unperturbed wave is directed along the $z$ axis $u_{g r 0}=c\left[\partial\left(N_{0} \omega_{0}\right) / \partial \omega_{0}\right]^{-1}=$ $c m_{0} /\left(m_{0}^{2}+m_{1}^{2}\right)^{-1}[5,15], c$ is the local propagation speed of small disturbances of the electron density irregularities.

The spectral density function $G(\mathbf{r}, v)$ is defined by

$$
\omega_{1}(\mathbf{r}, t)=\int_{-\infty}^{\infty} d v G(\mathbf{r}, v) \exp (i v t) .
$$

All quantities are assumed to be real functions of time $t$ and a three-dimensional spatial coordinate $\mathbf{r}$. Substitute this expression into (3). We suppose that randomly varying electron density irregularities having characteristic spatial scale $l$ are located in the slab $0<z<L_{0}$ and the fluctuations of the wave parameters are absent at $z<0$; at the observation point beyond the slab $z>L_{0}$ we get

$$
\begin{aligned}
& G(\mathbf{r}, v)=k_{0}\left(\Omega_{1}-i \Omega_{2}\right) \exp \left[-v \frac{m_{0}}{c}\left(i+\Omega_{0}\right) z\right] \\
& \int_{0}^{L_{0}} d \xi n_{1}(x, z, \xi, v) \exp \left[v \frac{m_{0}}{c}\left(i+\Omega_{0}\right) \xi\right] .
\end{aligned}
$$

It is evident that the instantaneous local frequency randomly varies in space and time, while these fluctuations at the observation point $\mathbf{r}$ and the moment $t$ are created due to multiple interaction of a wave with all irregularities laying on a path of wavy perturbations, and are determined by chaotical electron density fluctuations at the time moments when wavy perturbations pass through them with a group velocity.

For the second order statistical moments of the frequency fluctuations of the wave propagating distances $L_{0}>>l$ in a weakly absorptive plasma $\Omega_{0}<1$ we obtain:

$$
\begin{aligned}
& <\omega_{1} \omega_{1}^{*}>=\left(\Omega_{1}^{2}+\Omega_{2}^{2}\right) \frac{k_{0}^{2} c}{2 m_{0} \Omega_{0}} \int_{-\infty}^{\infty} d \mathbf{k}_{\perp} \int_{-\infty}^{\infty} d v v \\
& V_{n}\left(k_{x}, k_{y}, \frac{m_{0}}{c} v, v\right) \exp \left(-2 \Omega_{0} \frac{m_{0}}{c} v z\right) .
\end{aligned}
$$

$$
\begin{aligned}
& \cdot\left[\exp \left(2 \Omega_{0} \frac{m_{0}}{c} v L_{0}\right)-1\right] \\
& \frac{1}{2} \operatorname{Re}<\omega_{1} \omega_{1}>=\operatorname{Re} \frac{k_{0}^{2}}{2}\left(\Omega_{1}-i \Omega_{2}\right)^{2} L_{0} \int_{-\infty}^{\infty} d \mathbf{k}_{\perp} \int_{-\infty}^{\infty} d v v^{2} \\
& \cdot V_{n}\left(k_{x}, k_{y}, \frac{m_{0}}{c} v, v\right)
\end{aligned}
$$

where the pointed brackets indicate ensemble average, the asterisk complex conjugate, $\mathbf{k}_{\perp}=\left(k_{x}, k_{y}\right), V_{n}(\mathbf{k}, v)$ is an arbitrary correlation function of electron density fluctuations. For practical purposes it is usually convenient to assume that all processes under consideration are ergodic, as well as stationary, in time, in which case the statistical average can be regarded as a time average.

The variance of a real part of the frequency fluctuations describing the broadening of $t$ which are stationary in the wide sense, i.e., that the second order statistical moments are independent of $t$. The temporal spectrum is determined by the formula:

$$
\Delta=\Delta_{1}+\Delta_{2}
$$

Broadening of the temporal power spectrum $\Delta \equiv<\omega_{1}^{2}>/ \omega_{0}^{2}$ can be easily measured by experiment; $\Delta_{1} \equiv<\omega_{1} \omega_{1}^{*}>/ 2 \omega_{0}^{2}, \Delta_{2} \equiv \operatorname{Re}<\omega_{1} \omega_{1}>/ 2 \omega_{0}^{2}$. As the violation of the field coherence in medium with large-scale irregularities is caused by the phase fluctuations, we can suggest that the variance of the wave frequency $<\omega_{1}^{2}>$ keeps its sense at diffraction too. At those distances where the absorption becomes essential second term in (7) much less the first one.

\section{NUMERICAL CALCULATIONS}

The spectral density function which best describes the irregularities in the medium depends on the particular case and may differ from medium to medium. The most widely used spectral density function is the Gaussian, which has certain mathematical advantages.

At radio waves propagation in the ionosphere and cosmic plasma, where electron density irregularities are strongly aligned along the magnetic lines of forces, the influence of anisotropic irregularities on the statistical characteristics of scattered radio signals becomes very important. At the same time special attention is paid to the case when wave propagates at small angle $\gamma_{0}$ with respect to the elongated plasmonic irregularities. Anisotropic parameter $\chi=l_{\|} / l_{\perp}$ is the ratio of the longitudinal $l_{\|}$and transverse $l_{\perp}$ characteristic linear scales of electron density irregularities with respect to the external magnetic field; elongated plasmonic structures makes angle $\gamma_{0}$ to the homogeneous external magnetic field. In general case the anisotropy 
direction of plasmonic irregularities does not coincide with the direction of wave propagation.

If plasma moves along the homogeneous external magnetic field with constant velocity $V_{0}$, the spatialtemporal spectrum of electron density fluctuation has the anisotropic Gaussian form:

$$
\begin{aligned}
& V_{n}(\mathbf{k}, v)=\frac{\sigma_{n}^{2}}{16 \pi^{5 / 2}} \frac{l_{\|}^{3} T}{\chi^{2} a_{1}\left(1-e_{1}\right)^{1 / 2}} \exp \left[-\left(e_{2}+\frac{1}{p_{2} \chi^{2}}\right) .\right. \\
& \left.\frac{k_{y}^{2} l_{\|}^{2}}{4}-e_{3} \frac{k_{y} l_{\|}}{4} v T-e_{4} \frac{v^{2} T^{2}}{4}\right] \exp \left(-\frac{k_{x}^{2} l_{\|}^{2}}{4 \chi^{2}}-e_{5} \frac{k_{z}^{2} l_{\|}^{2}}{4}\right) \\
& \cdot \exp \left(-e_{6} k_{y} k_{z} l_{\|}^{2}+e_{7} k_{z} l_{\|} v T\right),
\end{aligned}
$$

where: $a_{1}=1+\frac{\eta^{2}}{p_{2}}, \eta=\frac{V_{0} T}{l_{\|}}, \quad p_{2}=\frac{\sin ^{2} \gamma_{0}+\chi^{2} \cos ^{2} \gamma_{0}}{\chi^{2}}$, $e_{1}=\frac{\eta}{2 a_{1}}, e_{2}=\frac{h_{1}^{2}}{a_{1}}+\frac{p_{2} h_{2}^{2}}{\left(1-e_{1}\right)^{2}}, h_{1}=\frac{\chi^{2}-1}{\chi^{2}} \frac{\eta}{p_{2}} \sin \gamma_{0} \cos \gamma_{0}$ $h_{2}=\frac{\left(\chi^{2}-1\right) \sin \gamma_{0} \cos \gamma_{0}}{\sin ^{2} \gamma_{0}+\chi^{2} \cos ^{2} \gamma_{0}}-\frac{\eta h_{1}}{a_{1} p_{2}}, h_{3}=\frac{\eta}{a_{1} p_{2}}$, $e_{3}=2\left[\frac{h_{1}}{a_{1}}-\frac{p_{2} h_{2} h_{3}}{\left(1-e_{1}\right)^{2}}\right], \quad e_{4}=\frac{1}{a_{1}}+\frac{p_{2} h_{3}^{2}}{\left(1-e_{1}\right)^{2}}$, $e_{5}=\frac{p_{2}}{\left(1-e_{1}\right)^{2}}, \quad e_{6}=\frac{h_{2} e_{5}}{2}, \quad e_{7}=\frac{h_{3} e_{5}}{2}, \quad T$ is the characteristic temporal scale of elongated plasmonic structures. If $V_{0}=0$ from (8) we obtain [18-20].

Rewrite (7) in nondimensional parameters, $v_{0}=1 / T$ is the frequency of the ionospheric irregularities. Substituting (8) into (5) and (6) we obtain second order statistical moments of scattered electromagnetic waves describing the broadening of the temporal spectrum along the external magnetic field:

$$
\begin{aligned}
& \Delta_{1}=\frac{\left(k_{0} l_{\|}\right)^{2}}{\chi^{2}} \frac{L_{0}}{l_{\|}}\left(\frac{v_{0}}{\omega_{0}}\right)^{2} \frac{\Omega_{1}^{2}+\Omega_{2}^{2}}{t_{1} t_{3}^{2} a_{1}\left(1-e_{1}\right)^{2}}\left\{\frac{z}{L_{0}}-\left(\frac{z}{L_{0}}-1\right)\right. \\
& \left.\cdot \exp \left[-4 \frac{m_{0} \Omega_{0}^{2}}{t_{3}^{2}}\left(\frac{L_{0}}{l_{\|}}\right)^{2}\left(\frac{v_{0}}{\omega_{0}}\right)^{2}\left(2 \frac{z}{L_{0}}-1\right)\right]\right\} \\
& \Delta_{2}=\frac{\left(k_{0} l_{\|}\right)^{2} \Omega_{1} \Omega_{2}}{t_{1} \chi^{2} a_{1}\left(1-e_{1}\right)^{1 / 2}}\left(\frac{v_{0}}{\omega_{0}}\right)^{2} \frac{L_{0}}{l_{\|}} \\
& \left(e_{4}-\frac{\zeta_{4}}{4 t_{1}^{2}}+4 \zeta_{1}-4 \zeta_{3}\right)^{-3 / 2}
\end{aligned}
$$

where: $t_{1}=\left(e_{2}+\frac{1}{p_{2} \chi^{2}}\right)^{1 / 2}, t_{2}=\frac{e_{3}}{4}+e_{6} m_{0} t_{0}$,

$$
\begin{aligned}
& t_{3}^{2}=e_{4}+e_{5} m_{0}^{2}\left(\frac{l_{\|}}{c T}\right)^{2}-4 e_{7} m_{0}\left(\frac{l_{\|}}{c T}\right)-4 \frac{t_{2}^{2}}{t_{1}^{2}}, t_{0}=\frac{l_{\|}}{c T}, \\
& Q=\left(e_{4}-\frac{\zeta_{4}}{4 t_{1}^{2}}+4 \zeta_{1}-4 \zeta_{3}\right)^{1 / 2}, \quad \zeta_{3}=e_{7} m_{0} t_{0}, \\
& \zeta_{1}=e_{5} \frac{m_{0}^{2}}{4} t_{0}^{2}, \quad \zeta_{2}=e_{6} m_{0} t_{0}, \quad \zeta_{4}=\left(e_{3}+4 \zeta_{2}\right)^{2} .
\end{aligned}
$$

The obtained results are valid for arbitrary propagation distance $z$. From the obtained result follow, that when dumping is small on the wavelength, but it is rather big on all length of the distance passed by a wave, frequency fluctuations grow much faster, than in the similar medium without absorption [5,15]. This growth continues also outside the layer containing irregularities (at $z>L_{0}$ ). It is essential to note that statistical moment (9) does not depend on a sign of the parameter $\Omega_{0}$, i.e., this is characteristic for both absorbing, and of active media. The reason of this effect is that, frequency perturbations in medium with complex refractive index not only are transferred along $\mathrm{z}$ axes with a group velocity [5], but also amplify. Obviously similar effect will take place also in a homogeneous medium with the complex refractive index for an incident frequency-modulated wave. The modulator in our case is a turbulent layer.

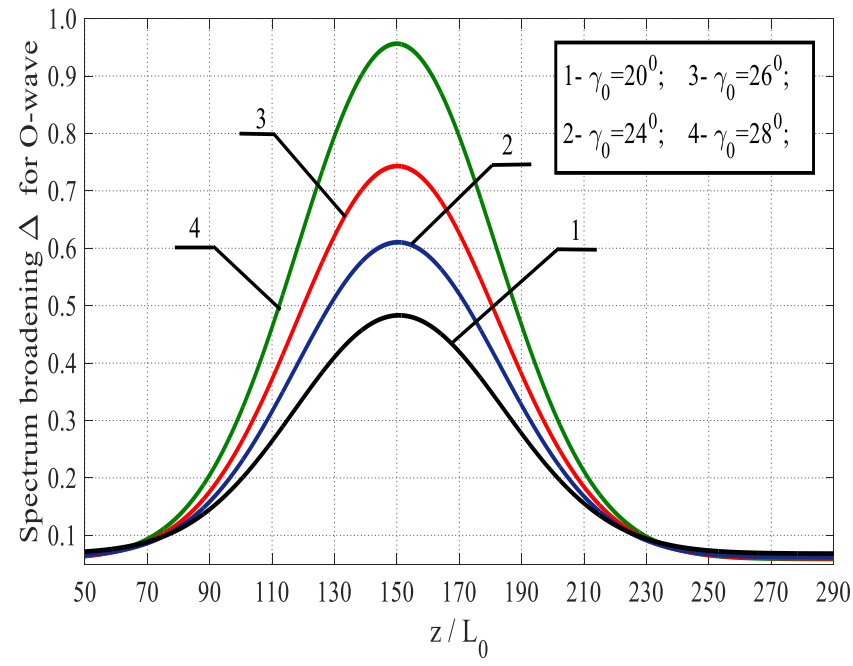

FIGURE 1. Broadening of the normzlized temporal spectrum of scattered ordinary electromagnetic waves vs - dimensionless dinstance parameter.

Numerical calculations are carried out for and incident wave with the frequency $3 \mathrm{MHz}$. Figure 1 shows plots of the broadening of the temporal power spectrum of a 
scattered ordinary electromagnetic waves in the polar region of the conductive magnetized turbulent plasma for the different inclination angles of elongated electron density irregularities with respect to the geomagnetic lines of forces in the interval $20^{0} \leq \gamma_{0} \leq 28^{0}$ at the anisotropic factor $\chi=15$.

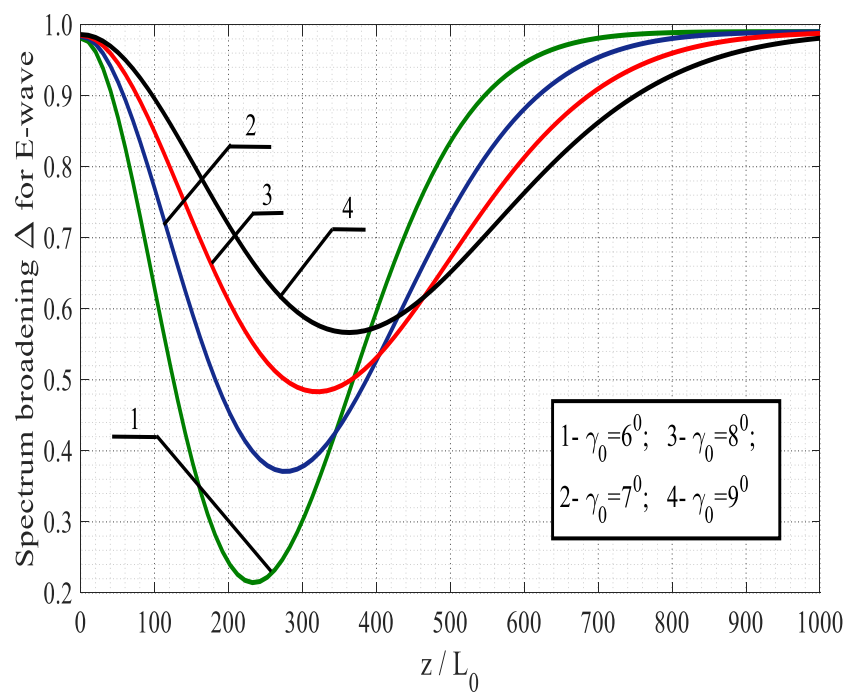

FFIGURE 2. The same as in Fig. 1, except that for scattered extraordinary electromagnetic wave.

Analyses show that the wave spectrum broadens with increasing strength of the randomness of the medium, and with increasing carrier frequency. The function $\Delta$ has a maximum at $\left(v_{0} / \omega_{0}\right)=10^{-4}$ and $z / L_{0}=150$ varying inclination angle $\gamma_{0}$; broadening of the temporal spectrum increases in proportion to the angle $\gamma_{0}$.

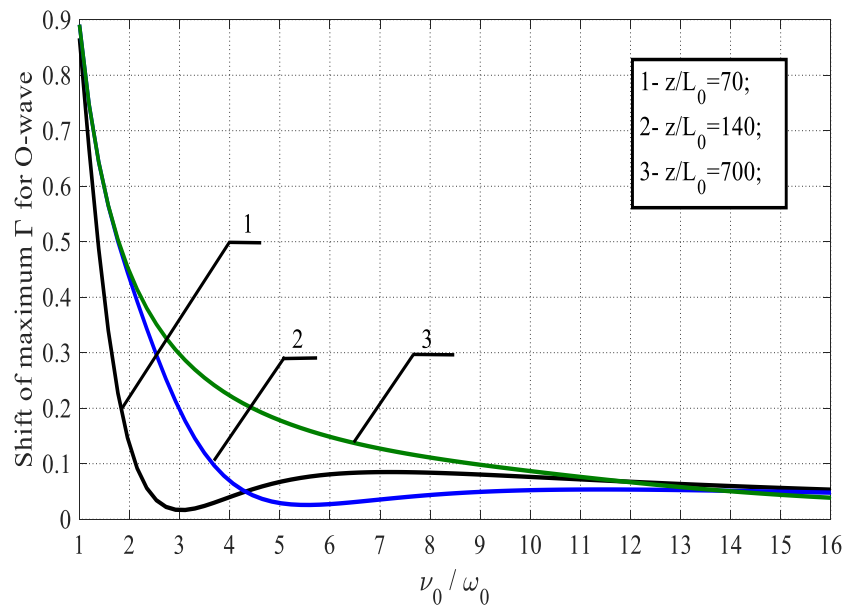

FIGURE 3. Shift of maximum of the temporal spectrum of the O-wave vs nondimension frequency parameter.
Figure 2 depicts the broadening of the temporal spectrum for scattered E-wave at $\left(v_{0} / \omega_{0}\right)=10$, varying inclination angle of elongated anisotropic plasmonic structures in the interval $6^{0} \leq \gamma_{0} \leq 9^{0}$. In this case temporal spectrum broadens in proportion of the angle $\gamma_{0}$; maximum of the spectrum is displaced: if $\gamma_{0}=6^{0}$ maximum is located at $\left(z / L_{0}\right)=234$, if $\gamma_{0}=9^{0}$ maximum of a curve shifts $\left(z / L_{0}\right)=446$.

Figure 3 illustrate shift of maximums of the temporal spectrum of the E-wave varying non-dimensional frequency parameter in the frequency band $2 \leq\left(v_{0} / \omega_{0}\right) \leq 16$.

Travelling distance by the wave outside a plasma slab minimum of the spectrum displaces: for the curve $\left(z / L_{0}\right)=70$, minimum is at $\left(v_{0} / \omega_{0}\right)=3.05$ and the broadening $\Delta=0.016$; for the curve $\left(z / L_{0}\right)=140$, we have minimum at $\left(v_{0} / \omega_{0}\right)=5.69$ and the broadening $\Delta=0.026$; starting from $\left(z / L_{0}\right)=700$ temporal spectrum not broadens. At large distances the spectral width approaches a limiting value.

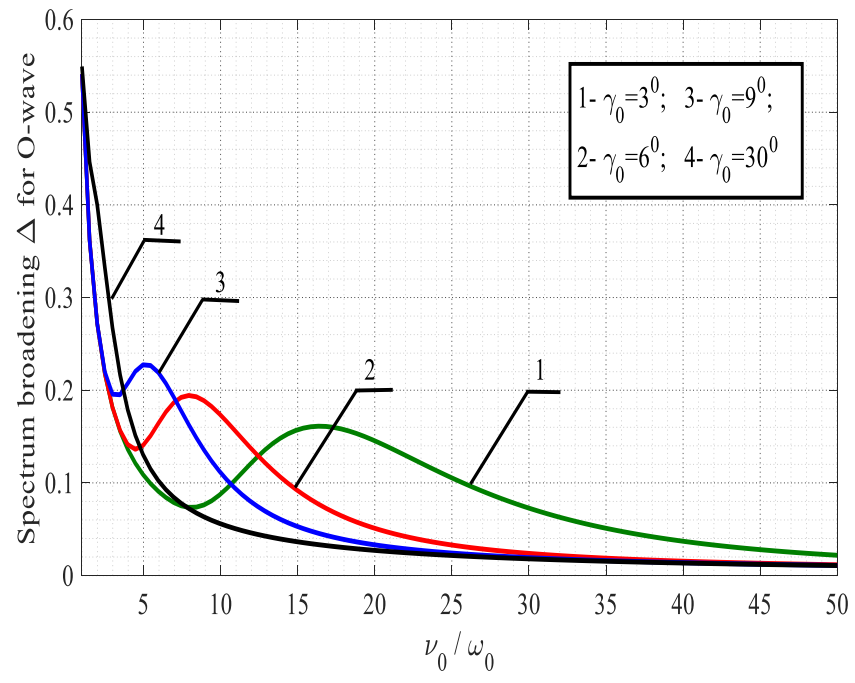

FIGURE 4. Broadening of the normalized temporal spectrum of scattered Owave as a function.

Curves describing the broadening of the temporal power spectrum of scattered O-wave at a distance $z / L_{0}=500$ are plotted in Fig. 4 for different inclination angle $\gamma_{0}$ of elongated ionospheric plasmonic structures. For $\gamma_{0}=3^{0}$, $\gamma_{0}=6^{0}, \gamma_{0}=9^{0}$ : local minimums are at $\left(v_{0} / \omega_{0}\right)=8.11$, $\left(v_{0} / \omega_{0}\right)=4.47$ and $\left(v_{0} / \omega_{0}\right)=3.23$, respectively; local maximums are at: $\left(v_{0} / \omega_{0}\right)=16.23,\left(v_{0} / \omega_{0}\right)=7.96$ and 
$\left(v_{0} / \omega_{0}\right)=4.88$, respectively. Decreasing inclination angle temporal power spectrum broadens.

Figure 5 illustrates shift of minimums of the temporal spectrum of the extraordinary wave as a function of the parameter $z / L_{0}$ at $\left(v_{0} / \omega_{0}\right)=10$, i.e., frequency of the

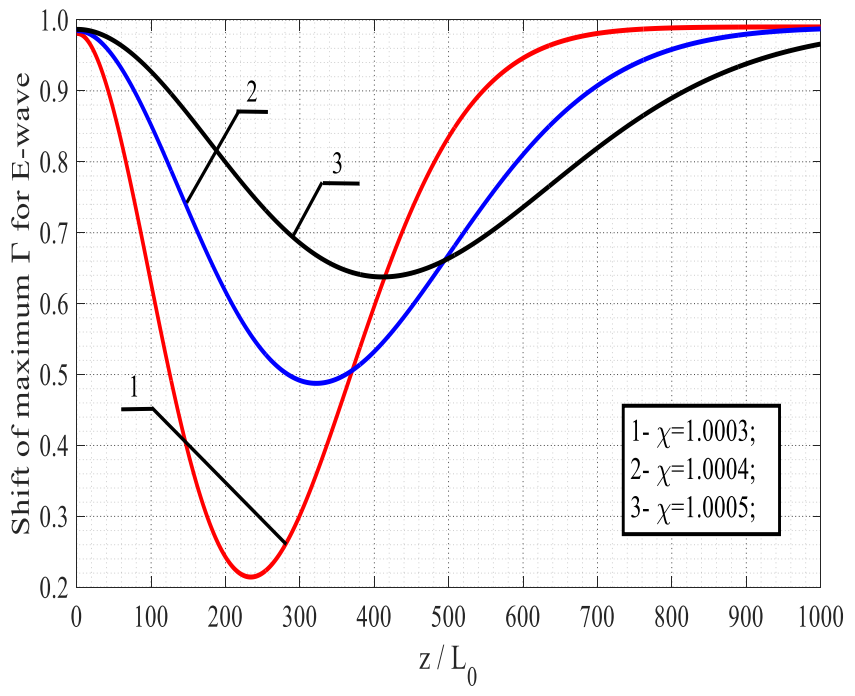

FIGURE 5. Displacement of the minimum of the temporal spectrum for Ewave as a function of distance.

temporal pulsation of electron density fluctuations exceeds ten times carrier frequency. In this case spectrum broadens and its maximum is displaced. At $\chi=1.003$, minimum of a curve is at $\left(z / L_{0}\right)=234$, at $\chi=1.005$, minimum is located at $\left(z / L_{0}\right)=411$. Temporal spectrum broadens two times.

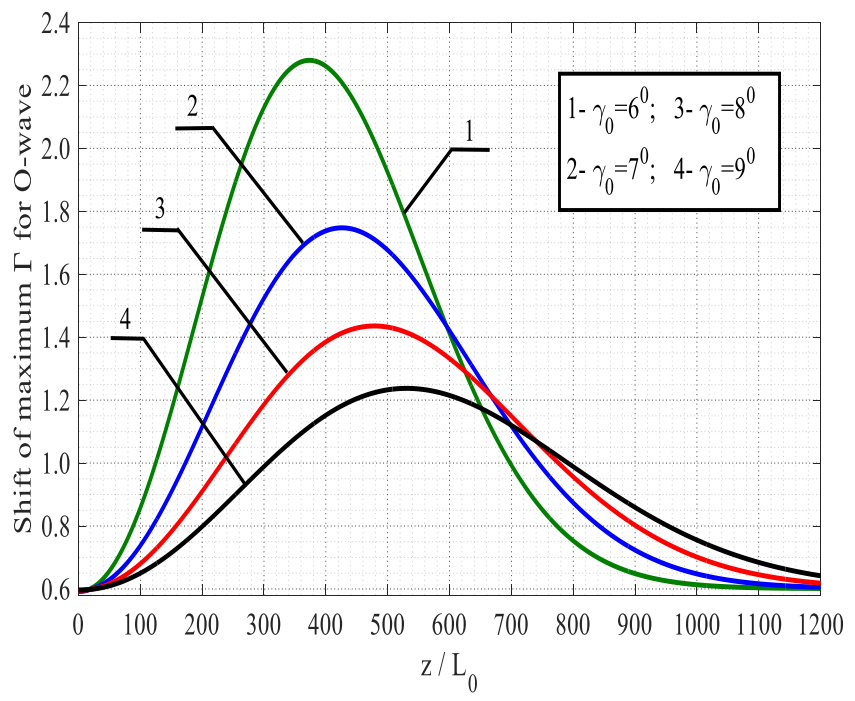

FIGURE 6. The same as in Fig. 5, except that for scattered O-wave.
Figure 6 depicts the broadening and the displacement of its maximum of scattered ordinary wave at small inclination angles $\gamma_{0}$ and $\left(v_{0} / \omega_{0}\right)=10$. At $\gamma_{0}=6$ maximum of a curve is at $\left(z / L_{0}\right)=373$, while at $\gamma_{0}=9^{0}$ maximum of a plot is located at $\left(z / L_{0}\right)=531$. Spectrum broadens approximately two times.

\section{CONCLUSION}

Transformation of the temporal spectrum of scattered ordinary and extraordinary waves caused by the anisotropic ionospheric components has been investigated for the first time on the basis of the stochastic transport differential equation for the frequency randomly varying in space and time. These statistical characteristics depends on the temporal pulsation of plasma parameters, propagation distance and propagation direction in the turbulent plasma; do not depend on an absorption sign and valid for both absorptive and active media. The obtained results are important in satellite communication and radar systems.

New features of the spectral bandwidth of radiowave signal will be revealed for different anisotropy parameters: the direction of an external magnetic field, plasma velocity, ionospheric conductivities, anisotropy factors and inclination angles of elongated plasmonic structures on different altitudes and in various regions of the terrestrial atmosphere.

The obtained results are applied to study propagation in space-time varying dielectrics, and plasma. Main effects of the broadening of the temporal spectrum are revealed at strong integrated absorption on a path and rather powerful source with high degree of monochromaticity is necessary for their experimental detection. The broadening of the spectrum is determined by the wave absorption not only in the nonstationary medium, but also on the subsequent section of a path. Concerning to the space radiation, nonstationarity of plasma of the stars and interstellar medium will be enough, if it is possible to analyze strongly weakened signal of monochromatic distant source caused due to absorption, that is now quite difficult. The problem is of importance since the performance of various existing and developing communication systems depends on the statistical properties of the signal after propagating through the transmission medium.

\section{REFERENCES}

[1] A.Ishimaru, Wave Propagation and Scattering in Random Media, Vol. 2, Multiple Scattering, Turbulence, Rough Surfaces and Remote Sensing, IEEE Press, Piscataway, New Jersey, USA, 1997.

[2] S. M. Rytov, Yu. A. Kravtsov, V.I. Tatarskii, Principles of Statistical Radiophysics. vol.4. Waves Propagation Through Random Media. Berlin, New York, Springer. 1989.

[3] Yu. A. Kravtsov, Yu. I. Orlov, Geometrical optics of Inhomogeneous Media. Moscow, Nauka, 1980 (in Russian).

[4] Yu. A. Kravtsov, Yu. A. Feizulin, A.G. Vinogradov, Radio Wave Propagation Through the Earth's Atmosphere, Radio and Communication, Moscow, 1983 (in Russian). 
[5] Yu. A. Kravtsov, L. A. Ostrovsky, N.S. Stepanov, "Geometrical Optics of Inhomogeneous and Nonstationary Dispersive Media," Proceedings IEEE, vol. 62, no 11, pp. 1492-1510, 1974.

[6] G. Jandieri, A. Ishimaru, B. Rawat, V. Gavrilenko, O. Kharshiladze, "Statistical Moments and Scintillation Level of Scattered Electromagnetic Waves in the Magnetized Plasma", Progress In Electromagnetics Research C, PIER C., vol. 84, pp. 11-22, 2018. DOI:10.2528/PIERC18030602

[7] G. Jandieri, A. Ishimaru, B. Rawat, N. Mchedlishvili, "Spatial Power Spectrum of Scattered Electromagnetic Waves in the Conductive Anisotropic Magnetized Plasma," Int. J. Microwave and Optical Technology, vol. 14, pp. 440-449, 2019. ISSN 1553-0396

[8] G. Jandieri, N. Zhukova, I. Jandieri, "Statistical Characteristics of Scattered Radiation in Medium with Spatial-Temporal Fluctuations of Electron Density and External Magnetic Field," J. Electromagnetic Analysis and Application, vol. 4, pp. 243-251, 2012. DOI: $10.4236 /$ jemaa.2012.46034.

[9] G. Jandieri, A. Ishimaru, B. Rawat, "Peculiarities of the Spatial Power Spectrum of Scattered Electromagnetic Waves in the Turbulent Collision Magnetized Plasma," Progress In Electromagnetics Research PIER, vol. 152, pp. 137-149, 2015. doi:10.2528/PIER15081802

[10] A. Ishimaru, "Wave beam and remote sensing" in Laser Beam Propagation in the Atmosphere, Ed. By J. W. Strohben, Chapter 5, pp. 169-213, Springer-Verlag, Berlin Heidelberg, New York, 1978.

[11] N. Kuznetsova, L. Chernov, "Allowance for the Doppler Effect in the Theory of Multiple Wave Scattering in a Randomly Inhomogeneous Medium," Sov. Phys. Acoust. vol. 23, pp. 93-94, 1977.

[12] V. Tatarskii, Wave Propagation in a Turbulent Medium. Dover, New York, 1967
[13] V. Gavrilenko, S. Petrov, "The features of the spectrum of electromagnetic waves multiply scattered in the turbulent plasma flow," Plasma Physics Report, vol. 12, \# 10, pp. 1257-1260, 1986.

[14] C. Liu, A. Wernik, K. Yeh, "Propagation of Pulse Trains through a random Medium," IEEE Trans. Antennas and Propagation, pp.624627,1974

[15] V. Gavrilenko, N. Stepanov, "Statistical characteristics of waves in the chaotically media with spatial-temporal irregularities," Izv. VUZ. Radiophysics, vol. 20, pp. 3-35, 1987.

[16] G. Jandieri, A. Ishimaru, N. Mchedlishvili, "Compensation Effect in the Conductive Auroral Regions of the Terrestrial Atmosphere," Progress In Electromagnetics Research M, PIER M, vol. 105, pp.119-129, 2021. DOI:10.2528/PIERM21081206

[17] V. Ginzburg, Propagation of Electromagnetic Waves in Plasma, Gordon and Beach, New York, 1961.

[18] G. Jandieri, "Double-Humped Effect" in the Turbulent Collision Magnetized Plasma," PIER M, vol. 48, pp. 95-102, 2016. doi:10.2528/PIERM16031203

[19] G. Jandieri, J. Diasamidze, I. Takidze, "Second Order Statistical Moments of the Phase Fluctuations of Scattered Radiation in the Collision Magnetized Plasma,' WORLDCOMP'16, CSC'16, pp.134$138,2016$.

[20] G. Jandieri, A. Ishimaru, V. Jandieri, A. Khantadze, Z. Diasamidze, "Model Computations of Angular Power Spectra for Anisotropic Absorptive Turbulent Magnetized Plasma," Progress In Electromagnetic Research, PIER 70, 307-328, 2007. DOI:10.2528/PIER07013103 